\title{
Pre-surgical Caregiver Burden and Anxiety Are Associated with Post-Surgery Cortisol over the Day in Caregivers of Coronary Artery Bypass Graft Surgery Patients
}

\author{
Claudio Singh Solorzano ${ }^{1}$ (D) $\cdot$ Andrew Steptoe ${ }^{1} \cdot$ Elizabeth Leigh $^{1} \cdot$ Tara Kidd $^{2} \cdot$ Marjan Jahangiri $^{3} \cdot$ Lydia Poole $^{1}$
}

Published online: 21 February 2019

(C) The Author(s) 2019

\begin{abstract}
Background The relationship between pre-surgical distress and diurnal cortisol following surgery has not been investigated prospectively in caregivers of coronary artery bypass graft $(\mathrm{CABG})$ patients before. We aimed to examine the relationship between pre-surgical anxiety and caregiver burden and diurnal cortisol measured 2 months after the surgery in the caregivers of CABG patients.

Method We used a sample of 103 caregivers of elective CABG patients that were assessed 28.86 days before and 60.94 days after patients' surgery. Anxiety and caregiver burden were assessed using the anxiety subscale of the Hospital Anxiety and Depression Scale and the Oberst Burden Scale respectively. Saliva samples were collected to measure cortisol area under the curve with respect to ground (AUCg) and diurnal cortisol slope. Anxiety and caregiver burden were entered into linear regression models simultaneously.

Results While high levels of pre-surgical anxiety were positively associated with increased follow-up levels of AUCg $(\beta=0.30$, $p=0.001)$, greater pre-surgery perceived burden score was associated with steeper cortisol slope $(\beta=0.27, p=0.017)$ after controlling for a wide range of covariates.

Conclusion These outcomes support the utility of psychological interventions aimed to increase the awareness of caregiving tasks and demands in informal caregivers.
\end{abstract}

\section{Introduction}

Informal caregiving for a sick relative or friend is a common experience in the UK, with estimates suggesting a 1-year prevalence of about $10 \%$ [1]. Caregiving for patients experiencing acute traumatic events such as coronary artery bypass graft (CABG) surgery often falls upon the spouse or partner of the patient. These caregivers have been found to experience disruption to their emotional and physical well-being. For example,

Claudio Singh Solorzano

c.solorzano@ucl.ac.uk

1 Department of Behavioural Science and Health, University College London, 1-19 Torrington Place, London WC1E 6BT, UK

2 School of Natural Sciences and Psychology, Liverpool John Moores University, Tom Reilly Building, Byrom Street, Liverpool L3 3AF, UK

3 Department of Cardiac Surgery, St George's Hospital, University of London, Blackshaw Road, London SW17 0QT, UK elevated anxiety [2] and an increase in caregiver burden following the surgery [3] are notable problems in the caregiving experience. A review on the impact of an acute cardiac event on patients' caregivers showed that caregivers are vulnerable to developing anxiety, depression, stress, caregiver burden and psychosocial and physical health problems [4]. Such burden has been associated with adverse physical health outcomes and premature mortality in caregivers [5]. The psychological and physical well-being of caregivers is not only important for their own health, but also for the effects on the patient's psychological adjustment and management of illness [6].

One hypothesised mediator of the association between caregiver anxiety and burden and poor health outcomes is via activation of the neuroendocrine axis. Indeed, chronic stress, such as caregiving, has long been associated with cortisol dysregulation [7]. Chronic stress is hypothesised to lead to a state of allostasis, the wear and tear on the body's stress system resulting from chronic overactivity or inactivity of physiological systems, including the hypothalamic-pituitaryadrenal (HPA) axis and resulting neuroendocrine dysregulation. Traditional caregiver studies have tended to look at 
caregivers of dementia patients in order to lend support to allostatic load theory [8-10]. This literature has expanded across the past decade to include research into non-elderly populations [11]. Moreover, other work has also studied the physiological consequences of caregiving for cancer patients [12]. However, whether the same pattern of findings is replicable in an acute caregiving situation, such as after CABG surgery, is currently less well understood.

Dysregulated cortisol has been associated with an array of poor health outcomes including an increased risk of all-cause and cardiovascular mortality in those with flatter diurnal slopes [13] and an increased risk of breast and lung cancer mortality again in those with flatter diurnal slopes $[14,15]$. Other aspects of the diurnal profile of cortisol have also been associated with deleterious effects for health. For example, greater cortisol output across the day has been associated with the extent of coronary atherosclerosis [16]. Therefore, there is a need for studies which attempt to better understand the causes of cortisol dysregulation in 'at risk' groups such as caregivers.

The aim of the current study was to investigate the prospective relationship between two markers of pre-surgical distress, namely anxiety and perceived caregiver burden, and cortisol output across the day measured 2 months after surgery in partners of CABG patients. Moreover, a secondary aim was to examine the independent effects of the two caregiver burden subscales: difficulty and time, on cortisol measured 2 months after surgery.

\section{Method}

\section{Participants}

Participants were the caregivers (spouse or co-habiting partner) of patients who took part in the ARCS (Adjustment and Recovery after Cardiac Surgery) study; full details are published elsewhere [17]. Of 142 recruited participants, 39 had missing data on covariates leaving an analytic sample of 103 caregivers of patients undergoing elective first-time CABG surgery at St George's Hospital, London; the 39 excluded caregivers did not differ from the analytic sample on any clinical or demographic variables. Cortisol area under the curve with respect to ground (AUCg) analyses had missing data on a small number of cases $(n=9)$, so separate $N$ s are reported for these analyses. Participants were included if they were over 18 years old, were fluent in English, and if they were the primary caregiver. On average, participants completed baseline assessments 28.86 days before patients' surgery and postsurgery assessment 60.94 days after the spouses' surgery. All procedures were carried out with written informed consent of the participant. The study was approved by the South West London research ethics committee.

\section{Measures}

\section{Independent Variables: Anxiety and Caregiver Burden}

Anxiety was assessed with the 7-items anxiety subscale of the Hospital Anxiety and Depression Scale (HADS) [18]. Participants were asked to rate the extent to which they had experienced each item (e.g. "worrying thoughts go through my mind') in the previous 2 weeks. This scale has been shown to be a reliable measure of anxiety screening in the general population [19] with higher scores indicating greater anxiety (ranging from 0 to 21). The HADS questionnaire has previously been administered in studies of CABG caregivers [2] and in caregivers of patients undergoing cardiac surgery [20]. This subscale was used as a continuous variable (Cronbach's alpha $=0.88$ ).

Caregiver burden was measured using the 15-item Oberst Burden Scale (OCBS) [21], in which each caregiving task (e.g. emotional support, 'being there' for your partners) was evaluated with a 5-point Likert scale across two dimensions: difficulty and time. Each item is answered with reference to the question 'How much time do you spend and how difficult is each activity for you to do?' This scale was selected because it has been used almost exclusively in partners of patients undergoing CABG surgery $[3,22,23]$. It is considered suitable for use in cardiac patients' partners as it assesses the burden related to everyday activities, which are necessary for the selfmanagement of cardiac illness, covering a wide range of practical and personal care tasks. The total burden score was obtained by calculating the square root of the product of the two subscales (Cronbach's alpha $=0.93$ ). Both subscales and total score could range from 15 to 75 , with higher scores indicating greater burden. In further analyses to look at the burden change between baseline and follow-up, the total burden score was split at the 75th percentile (23.87) to create low and high burden subgroups. For illustration purposes, we also divided caregiving difficulty in the same manner (75th percentile $=17.00$ ) to create low and high caregiving difficulty groups.

\section{Dependent Variables: Cortisol AUCg and Cortisol Slope}

All details of the saliva collection protocol are provided elsewhere [17]. Briefly, participants' pre- and post-surgery saliva samples were collected at 7 time points over the course of a single day (on waking, $30 \mathrm{~min}$ after awakening, $10 \mathrm{am}$, noon, $4 \mathrm{pm}, 8 \mathrm{pm}$ and bedtime) using Salivettes (Sarstedt, UK). On average, the samples were obtained 28.86 days before and 60.94 days after patients' surgery; tubes were returned by post and were stored at $-20^{\circ} \mathrm{C}$ for batch analysis at a later date by the University of Dresden (Germany) using a time-resolved immunoassay with fluorescence detection. Cortisol AUCg was derived from the trapezoid formula [24], with higher values indicating greater cortisol output across the day. Cortisol slope over the day was calculated as the reduction 
of cortisol per hour $(\mathrm{nmol} / \mathrm{L} / \mathrm{h})$ using regression on cortisol values, excluding the $30 \mathrm{~min}$ after wakening sample [25]. Slope values closer to zero reflect flatter diurnal rhythms.

\section{Covariates}

Caregivers' age, gender, number of people in the household (including the participant), occupation and smoking were selfreported at baseline, while patients' clinical risk was assessed using the EuroSCORE (European System for Cardiac Operative Risk Evaluation) index [26]. Items were scored using the 'logistic EuroSCORE' method to generate a percentage mortality risk estimate. Caregivers listed their current occupation, which was classified according to the Office of National Statistics Standard Occupation Classification (SOC) 2010 index [27]. Participants were subsequently categorised into 9 groups, from high to low occupation, using $\mathrm{SOC}$ as an indicator of socioeconomic status (SES). Smoking was measured as a binary variable (smoker/non-smoker).

\section{Statistical Analysis}

All outliers greater than 3 standard deviations (SD) from the mean were removed from the cortisol data. Cortisol data were normally distributed, so the raw scores are reported here. Change over time of cortisol AUCg and slope were assessed using paired $t$ tests, which was also used to compare the change of burden from baseline to follow-up in those who at baseline were either in the high or low total burden group. Multiple linear regression was used to model the association between pre-surgery anxiety and caregiver burden and cortisol $\mathrm{AUCg}$ and slope values. Anxiety and caregiver burden were entered into models simultaneously in order to assess their independent effects. Similar findings were found entering caregiver burden and anxiety symptoms separately into models and so are not reported here. In both models, age, sex, SES, number in the household, smoking, patients' EuroSCORE and baseline of the cortisol-dependent variable were entered as covariates. Given the large proportion of women in our study, secondary analyses were performed by entering mean-centred interaction terms into the models to examine the sex interaction with anxiety and caregiver burden. In further analyses, baseline caregiver burden was replaced by baseline caregiving difficulty and time subscales in fully adjusted regression models. Variance inflation factor (VIF) values and tolerance values were generated for all regression models to assess multicollinearity and the assumption was non violated (VIF $<10$ and tolerance $>0.1$ ). All data analyses were conducted using IBM SPSS Statistics version 22 (SPSS Inc.).

\section{Results}

Demographic characteristics for all participants who completed baseline and follow-up are presented in Table 1. On average, anxiety levels significantly decreased over time (baseline: mean 6.39, SD 4.37; follow-up: mean 5.27, SD 3.26; $t=3.12$, $p=0.002$ ), while caregiver burden scores had a significant increase following the surgery (baseline: mean 21.66, SD 5.90; follow-up: mean 26.21, SD 7.76; $t=-6.89, p<0.001$ ). When we split the file by low/high baseline total burden, the low total burden group had a significant rise in burden from baseline to follow-up ( $t=-8.20, p<0.001)$, while the high total burden group did not $(t=-1.13, p=0.269)$. Anxiety and caregiver burden at pre-surgical baseline were moderately correlated $(r=0.40, p<0.001)$.

Caregivers' cortisol output over the day (AUCg) were higher before patients' surgery compared with follow-up ( $t=1.46, p=0.148)$, while baseline cortisol slope was slightly flatter compared with post-surgery slope values $(t=-1.41$, $p=0.163)$. Nevertheless, these changes were non-significant.

Table 1 Demographic, biological and psychological characteristics of the sample $(N=103)$

\begin{tabular}{|c|c|}
\hline Characteristic & Mean $\pm \mathrm{SD}$ or $N(\%)$ \\
\hline \multicolumn{2}{|l|}{ Baseline demographic variables } \\
\hline Age & $66.15 \pm 8.31$ \\
\hline Sex - Female & $99(96.1)$ \\
\hline \multicolumn{2}{|l|}{ Occupation classification } \\
\hline High & $38(36.9)$ \\
\hline Intermediate & $39(37.9)$ \\
\hline Low & $26(25.2)$ \\
\hline Household of two persons & $92(89.3)$ \\
\hline Smoker & $3(2.9)$ \\
\hline \multicolumn{2}{|l|}{ Cortisol variables } \\
\hline Cortisol $\mathrm{AUC}_{\mathrm{g}}^{\mathrm{a}}$-baseline $(\mathrm{nmol} / \mathrm{L})^{*}$ & $7853.56 \pm 2531.22$ \\
\hline Cortisol AUC ${ }_{\mathrm{g}}{ }^{\mathrm{a}}$-follow-up (nmol/L)* & $7479.31 \pm 2407.91$ \\
\hline Cortisol slope-baseline $(\mathrm{nmol} / \mathrm{L} / \mathrm{h})$ & $0.016 \pm 0.010$ \\
\hline Cortisol slope-follow-up (nmol/L/h) & $0.018 \pm 0.009$ \\
\hline \multicolumn{2}{|l|}{ Baseline psychological distress } \\
\hline Anxiety $^{\mathrm{b}}$ & $6.39 \pm 4.37$ \\
\hline Total caregiver burden ${ }^{\mathrm{c}}$ & $21.66 \pm 5.90$ \\
\hline Caregiving time $\mathrm{e}^{\mathrm{c}}$ & $27.25 \pm 8.90$ \\
\hline Caregiving difficulty ${ }^{\mathrm{c}}$ & $17.40 \pm 4.76$ \\
\hline \multicolumn{2}{|l|}{ Patients' clinical variable } \\
\hline EuroSCORE $(\%)^{\mathrm{d}}$ & $4.25 \pm 3.55$ \\
\hline \multicolumn{2}{|l|}{${ }^{*} N=94$} \\
\hline \multicolumn{2}{|c|}{${ }^{a}$ Cortisol area under the curve with respect to the ground } \\
\hline \multicolumn{2}{|c|}{${ }^{\mathrm{b}}$ Anxiety subscale of HADS (Hospital Anxiety Depression Scale) } \\
\hline \multicolumn{2}{|c|}{${ }^{\mathrm{c}}$ OBS (Oberst Burden Scale) } \\
\hline \multicolumn{2}{|c|}{${ }^{\mathrm{d}}$ European System for Cardiac Operative Risk Evaluation } \\
\hline
\end{tabular}


Regression analyses showed that caregiver anxiety levels at pre-surgery baseline were associated with cortisol AUCg following surgery $(\beta=0.29, p=0.001)$, while caregiver burden did not $(\beta=-0.02, p=0.786)$, after controlling for all covariates. Baseline cortisol AUCg $(\beta=0.49, p<0.001)$ and gender ( $\beta=0.20, p=0.018$ ) were also significantly positively associated with cortisol AUCg cortisol post-surgery follow-up. The model accounted for $44 \%$ of variance in cortisol AUCg. See Table 2 for these results.

In a separate model (See Table 3), after controlling for covariates, pre-surgery caregiver burden was significantly associated with cortisol slope post-surgery $(\beta=0.27, p=0.017)$, but baseline anxiety was not $(\beta=-0.05, p=0.630)$. Greater pre-surgical burden was associated with steeper cortisol decline over the day. The baseline values of cortisol slope were positively associated with cortisol slope at follow-up ( $\beta=$ $0.28, p=0.008)$. There were no other significant variables. The model accounted for $14 \%$ of variance in cortisol slope.

In secondary analyses, we examined the sex $\times$ anxiety interaction by entering the mean-centred interaction term in models to predict cortisol AUCg. There was no significant interaction $(p=0.983)$. We also examined the sex $\times$ burden interaction by entering the mean-centred interaction term in models to predict cortisol slope. Again, there was no significant interaction $(p=0.208)$.

In further analyses using the caregiver burden subscales (caregiving time and caregiving difficulty), pre-surgical caregiving difficulty $(\beta=0.328, p=0.016)$, but not time $(\beta=$ $-0.020, p=0.877$ ), was associated with cortisol slope post-surgery. The relationship between post-surgery cortisol slope and baseline caregiving difficulty values is illustrated in Fig. 1.

\section{Discussion}

We found that higher pre-surgical anxiety was associated with greater post-surgery cortisol AUCg and that increased caregiver burden at baseline was associated with a steeper cortisol slope at follow-up. These associations were independent of all covariates included in the models and of the other distress marker, since anxiety and caregiver burden were entered simultaneously into regression models. These are the first findings to our knowledge that have demonstrated the effects of pre-surgery distress on post-surgery cortisol response in caregivers of $C A B G$ patients, suggesting the importance of providing support to partners who take on acute caregiving roles.

In the first prospective model, we found that greater anxiety, but not burden, prior to the patient's surgery was associated with greater post-surgery cortisol AUCg. These findings lend support to allostatic load theory in caregivers [8] and are further supported by previous research which has found an effect of anxiety on greater cortisol AUC in cross-sectional analyses [28]. Our study was able to explore longitudinal effects, suggesting pre-surgical anxiety may have a detrimental effect on caregivers' cortisol regulation up to 2 months after the CABG surgery.

Perhaps less intuitively we also found that greater presurgical caregiving burden, but not anxiety, was associated with a steeper slope at the post-surgery follow-up. A steeper slope is usually considered to be adaptive [29], so our finding is in opposition to what we might expect based on allostatic load theory. However, we propose that this finding can be explained by the caregiving situation whereby the baseline measurement of caregiver burden indicates a readiness to
Table 2 Multiple regression on baseline caregiver psychological distress predicting cortisol $\mathrm{AUCg}$ $(N=94)$

\begin{tabular}{|c|c|c|c|c|c|}
\hline Model & $B$ & SE & $95 \% \mathrm{CI}$ & $\beta$ & $p$ \\
\hline \multicolumn{6}{|l|}{ Step 1} \\
\hline Total caregiver burden ${ }^{\mathrm{a}}$ & 23.176 & 43.455 & {$[-63.143 ; 109.495]$} & 0.057 & 0.595 \\
\hline Anxiety $^{\mathrm{b}}$ & 160.433 & 60.598 & {$[40.063 ; 280.802]$} & 0.282 & 0.010 \\
\hline \multicolumn{6}{|l|}{ Step 2} \\
\hline Total caregiver burden ${ }^{a}$ & -10.252 & 37.575 & {$[-84.973 ; 64.470]$} & -0.025 & 0.786 \\
\hline Anxiety ${ }^{\mathrm{b}}$ & 167.074 & 50.497 & {$[66.655 ; 267.494]$} & 0.294 & 0.001 \\
\hline Age & -11.856 & 33.555 & {$[-78.585 ; 54.872]$} & -0.038 & 0.725 \\
\hline Gender & 2762.889 & 1145.771 & [484.398; 5041.379] & 0.203 & 0.018 \\
\hline Number in household & 553.923 & 408.506 & {$[-258.436 ; 1366.282]$} & 0.126 & 0.179 \\
\hline Occupation & -141.418 & 106.912 & {$[-354.024 ; 71.188]$} & -0.112 & 0.190 \\
\hline Smoking & -2082.981 & 1192.525 & {$[-4454.447 ; 288.485]$} & -0.153 & 0.084 \\
\hline Patients' EuroSCORE ${ }^{\mathrm{c}}$ & 92.978 & 62.721 & {$[-31.751 ; 217.706]$} & 0.142 & 0.142 \\
\hline Baseline cortisol $\mathrm{AUCg}^{\mathrm{d}}$ & 0.471 & 0.086 & {$[0.300 ; 0.642]$} & 0.496 & $<0.001$ \\
\hline
\end{tabular}


Table 3 Multiple regression on baseline caregiver psychological distress predicting cortisol slope $(N=103)$

\begin{tabular}{lcllrl}
\hline Model & $B$ & SE & $95 \%$ CI & $\beta$ & $p$ \\
\hline $\begin{array}{l}\text { Step 1 } \\
\text { Total caregiver burden }\end{array}$ & 0.0004 & 0.0002 & {$[0.00007,0.001]$} & 0.258 & 0.017 \\
$\begin{array}{l}\text { Anxiety } \\
\text { Step 2 }\end{array}$ & -0.0002 & 0.0002 & {$[-0.001 ; 0.0002]$} & -0.103 & 0.334 \\
Total caregiver burden & \\
$\quad$ Anxiety & & & & & \\
Age & 0.0004 & 0.0002 & {$[0.0001 ; 0.001]$} & 0.272 & 0.017 \\
Gender & -0.0001 & 0.0002 & {$[-0.001 ; 0.0003]$} & -0.052 & 0.630 \\
Number in household & 0.0001 & 0.005 & {$[-0.009 ; 0.010]$} & 0.002 & 0.982 \\
Occupation & 0.002 & 0.002 & {$[-0.001 ; 0.006]$} & 0.131 & 0.249 \\
Smoking & 0.0004 & 0.0005 & {$[-0.001 ; 0.001]$} & -0.080 & 0.427 \\
Patients' EuroSCORE & & & & \\
Baseline cortisol slope & 0.002 & 0.005 & {$[-0.009 ; 0.013]$} & 0.040 & 0.689 \\
\hline
\end{tabular}

${ }^{\text {a }}$ OBS (Oberst Burden Scale)

${ }^{\mathrm{b}}$ Anxiety subscale of HADS (Hospital Anxiety Depression Scale)

${ }^{\mathrm{c}}$ European System for Cardiac Operative Risk Evaluation provide care following patient's surgery. In this scenario, higher pre-surgical caregiver burden indicates a greater aptitude to meet the additional caregiving requirements following surgery. Support for this hypothesis is lent by the fact we observed different increases in burden over time when we split our file by baseline total burden. Caregivers who had a high level of burden at baseline did not show a significant increase in burden at follow-up, while the low baseline burden group did. Previous findings have shown that increased anticipatory stress before a psychosocial stressor predicted a stepper cortisol recovery following a laboratory stress test [30]. These authors explained this result as a possible healthy effect of anticipatory stress inducing high vigilance and a readiness to respond to possible subsequent stressors. However, this hypothesis requires further testing in future studies.

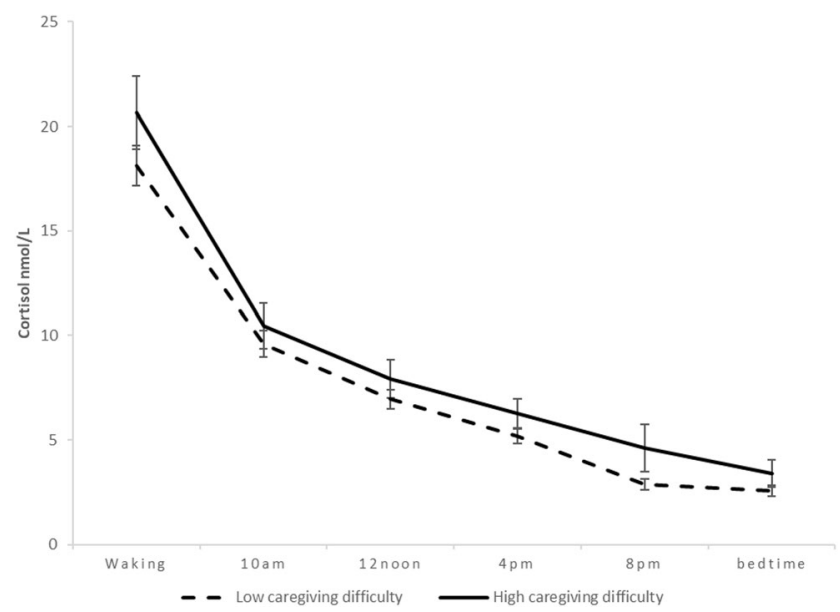

Fig. 1 Diurnal cortisol output of caregivers 60.94 days following CABG surgery by baseline levels of caregiving difficulty. Bars indicate standard errors of the mean $(N=103)$
A meta-analysis of psychosocial interventions for patients and their informal caregivers showed overall positive effects for outcomes including depression and anxiety [31]. However, there is a lack of research investigating the effects of interventions in caregivers of acutely ill patients such as those in our CABG surgery sample. This has been attributed to reasons such as a lack of resources, interest and knowledge among staff, and practical and psychological barriers among patients and partners [32]. Spouses and partners of CABG surgery patients are often faced with uncertainty about the responsibilities of the caregiving role following the patients' discharge which contribute to anxiety, burden and worries about their preparedness for responding properly to patient demands [33]. A systematic review has revealed that a modest number of intervention studies $(N=7)$ targeting coronary heart disease caregivers' well-being have shown trends toward improvements in anxiety, knowledge and satisfaction in care [34]. The VITAL telehealth programme used a randomised controlled trial design to investigate the effects of a telephone intervention on CABG patients and their caregiving partners' psychological state in the period following hospital discharge. This study demonstrated a positive effect of the intervention on anxiety and depression symptoms [35]. Such work indicates the potential benefits of intervening with this population, although further research is needed to examine the long-term effects of such trials.

Our study has a number of strengths. First, we used a longitudinal design so were able to demonstrate a prospective relationship over time. Moreover, cortisol was measured at different time points across the day allowing us to get a reliable cortisol profile. However, limitations include our reliance on questionnaire measures to assess anxiety and burden symptoms, which restricts us from generalising our results to 
clinical samples. Furthermore, our sample was composed mainly of female participants. This prevalence is explained by the predominance of men in the UK CABG surgical population [36]; however, our findings may not be readily generalisable to male caregivers. Lastly, both pre- and postsurgery cortisol was assessed only on a single day.

In conclusion, greater anxiety in caregivers 1 month before patients' surgery was associated with higher cortisol output across the day (AUCg) at 2-months follow-up, while increased pre-surgical level of caregiver burden was significantly associated with a steeper cortisol slope following patients' surgery. More research is needed to test the types of interventions that would be most acceptable and efficacious in this kind of caregivers.

Funding This project was funded by the British Heart Foundation (RG/ 10/005/28296).

\section{Compliance with Ethical Standards}

Ethical Approval and Informed Consent All procedures performed in studies involving human participants were in accordance with the ethical standards of the responsible committee on human experimentation (institutional and national) and with the Helsinki Declaration of 1975, as revised in 2000. Informed consent was obtained from all participants for being included in the study.

Conflict of Interest The authors declare that they have no conflict of interest.

Open Access This article is distributed under the terms of the Creative Commons Attribution 4.0 International License (http:// creativecommons.org/licenses/by/4.0/), which permits unrestricted use, distribution, and reproduction in any medium, provided you give appropriate credit to the original author(s) and the source, provide a link to the Creative Commons license, and indicate if changes were made.

Publisher's Note Springer Nature remains neutral with regard to jurisdictional claims in published maps and institutional affiliations.

\section{References}

1. White C. 2011 Census analysis : unpaid care in England and Wales, 2011 and comparison with 2001. 2013;1-29.

2. Davies N. Carers' opinions and emotional responses following cardiac surgery: cardiac rehabilitation implications for critical care nurses. Intensive Crit Care Nurs. 2000;16:66-75.

3. Stolarik A, Lindsay P, Sherrard H, Woodend AK. Determination of the burden of care in families of cardiac surgery patients. Prog Cardiovasc Nurs. 2000;15:4-10.

4. Randall G, Molloy GJ, Steptoe A. The impact of an acute cardiac event on the partners of patients: a systematic review. Health Psychol. Rev. 2009.

5. Schulz R, Beach SR. Caregiving as a risk factor for mortality. Am Med Assoc. 1999;282:2215-9.

6. Moser DK, Dracup K. Role of spousal anxiety and depression in patients' psychosocial recovery after a cardiac event. Psychosom Med. 2004;66:527-32.
7. Vedhara K, Cox NKM, Wilcock GK, Perks P, Hunt M, Anderson S, et al. Chronic stress in elderly carers of dementia patients and antibody response to influenza vaccination. Lancet. 1999;353:627-31.

8. Bennett JM, Fagundes CP, Kiecolt-Glaser JK. The chronic stress of caregiving accelerates the natural aging of the immune system. Immunosenescence Psychosoc Behav Determ. New York, NY: Springer New York; 2013. p. 35-46.

9. Allen AP, Curran EA, Duggan Á, Cryan JF, Chorcoráin AN, Dinan TG, et al. A systematic review of the psychobiological burden of informal caregiving for patients with dementia: focus on cognitive and biological markers of chronic stress. Neurosci. Biobehav. Rev. 2017. p. 123-64.

10. Watson B, Tatangelo G, McCabe M. Depression and anxiety among partner and offspring carers of people with dementia: a systematic review. Gerontologist. 2018.

11. Bevans M, Sternberg EM. Caregiving burden, stress, and health effects among family caregivers of adult cancer patients. JAMA J Am Med Assoc. 2012;307:398-403.

12. Rohleder N, Marin TJ, Ma R, Miller GE. Biologic cost of caring for a cancer patient: dysregulation of pro- and anti-inflammatory signaling pathways. J Clin Oncol American Society of Clinical Oncology. 2009;27:2909-15.

13. Kumari M, Shipley M, Stafford M, Kivimaki M. Association of diurnal patterns in salivary cortisol with all-cause and cardiovascular mortality: findings from the Whitehall II study. J Clin Endocrinol Metab. 2011;96:1478-85.

14. Sephton SE. Diurnal cortisol rhythm as a predictor of breast cancer survival. J Natl Cancer Inst. 2000;92:994-1000.

15. Sephton SE, Lush E, Dedert EA, Floyd AR, Rebholz WN, Dhabhar FS, et al. Diurnal cortisol rhythm as a predictor of lung cancer survival. Brain Behav Immun. 2013;30:S163-70.

16. Dekker MJHJ, Koper JW, Van Aken MO, Pols HAP, Hofman A, De Jong FH, et al. Salivary cortisol is related to atherosclerosis of carotid arteries. J Clin Endocrinol Metab. 2008;93:3741-7.

17. Ronaldson A, Kidd T, Poole L, Leigh E, Jahangiri M, Steptoe A. Diurnal cortisol rhythm is associated with adverse cardiac events and mortality in coronary artery bypass patients. J Clin Endocrinol Metab. 2015;100:3676-82.

18. Zigmond AS, Snaith RP. The hospital anxiety and depression scale. Acta Psychiatr Scand. 1983;67:361-70.

19. Bjelland I, Dahl AA, Haug TT, Neckelmann D. The validity of the hospital anxiety and depression scale. J Psychosom Res. 2002;52:69-77.

20. Young E, Eddleston J, Ingleby S, Streets J, McJanet L, Wang M, et al. Returning home after intensive care: a comparison of symptoms of anxiety and depression in ICU and elective cardiac surgery patients and their relatives. Intensive Care Med Springer-Verlag. 2005;31:86-91.

21. Oberst MT, Thomas SE, Gass KA, Ward SE. Caregiving demands and appraisal of stress among family caregivers. Cancer Nurs. 1989. p. 209-15.

22. Halm MA, Treat-Jacobson D, Lindquist R, Savik K. Correlates of caregiver burden after coronary artery bypass surgery. Nurs Res. 2006;55:426-36.

23. Halm MA, Treat-Jacobson D, Lindquist R, Savik K. Caregiver burden and outcomes of caregiving of spouses of patients who undergo coronary artery bypass graft surgery. Heart Lung. 2007;36:170-87.

24. Pruessner JC, Kirschbaum C, Meinlschmid G, Hellhammer DH. Two formulas for computation of the area under the curve represent measures of total hormone concentration versus time-dependent change. Psychoneuroendocrinology. 2003;28:916-31.

25. Messerli-Bürgy N, Molloy GJ, Wikman A, Perkins-Porras L, Randall $\mathrm{G}$, Steptoe A. Cortisol levels and history of depression in acute coronary syndrome patients. Psychol Med. 2012;42:1815-23. 
26. Roques F, Michel P, Goldstone AR, Nashef SAM. The logistic EuroSCORE. Eur Heart J. 2003;24:1-2.

27. Office for National Statistics. Standard Occupational Clasification 2010: Volume 1 Structure and descriptions of unit groups. 2010. http://www.ons.gov.uk/ons/guide-method/classifications/currentstandard-classifications/soc2010/soc2010-volume-1-structure-anddescriptions-of-unit-groups/soc2010-volume-1.pdf

28. Mantella RC, Butters MA, Amico JA, Mazumdar S, Rollman BL, Begley AE, et al. Salivary cortisol is associated with diagnosis and severity of late-life generalized anxiety disorder. Psychoneuroendocrinology. 2008;33:773-81.

29. Adam EK, Kumari M. Assessing salivary cortisol in large-scale, epidemiological research. Psychoneuroendocrinology. 2009;34: 1423-36.

30. Juster RP, Perna A, Marin MF, Sindi S, Lupien SJ. Timing is everything: anticipatory stress dynamics among cortisol and blood pressure reactivity and recovery in healthy adults. Stress. 2012;15: 569-77.

31. Martire LM, Lustig AP, Schulz R, Miller GE, Helgeson VS. Is it beneficial to involve a family member? A meta-analysis of psychosocial interventions for chronic illness. Heal. Psychol. 2004. p. 599-611.
32. Nissen NK, Kjøller M, Madsen M, Waldorff SB, Olsen Zwisler AD. Are hospitals also for relatives? A survey of hospitals' activities regarding relatives of cardiac patients. Scand J Public Health. 2008;36:827-31.

33. Halm MA. Specific needs, concerns, strategies and advice of caregivers after coronary artery bypass surgery. Hear Lung J Acute Crit Care. 2016;45:416-22.

34. Reid J, Ski CF, Thompson DR. Psychological interventions for patients with coronary heart disease and their partners: a systematic review. PLoS One. 2013;8:e73459.

35. Keeping-Burke L, Purden M, Frasure-Smith N, Cossette S, Mccarthy F, Amsel R. Bridging the transition from hospital to home: effects of the VITAL telehealth program on recovery for CABG surgery patients and their caregivers. Res Nurs Health. 2013;36:540-53.

36. Poole L, Kidd T, Leigh E, Ronaldson A, Jahangiri M, Steptoe A. Depression, C-reactive protein and length of post-operative hospital stay in coronary artery bypass graft surgery patients. Brain Behav Immun. 2014;37:115-21. 